\title{
La pertinencia en la aplicación de estrategias de comprensión lectora y el nivel educativo de los docentes
}

Alma Y. Aguilar Zedeño, ${ }^{1}$ María Teresa Dávalos Romo, ${ }^{1}$ Jennifer Rentería Conseción ${ }^{1}$ y Aidé T. Vital Caballero ${ }^{1}$

\section{Introducción}

La formación de los docentes mexicanos ha sido un tema necesario de ser entendido y analizado en el país, debido a las múltiples críticas que se han arrojado a partir de evaluaciones como ENLACE O PISA, las cuales han mostrado que el docente, sobre todo de nivel básico, presenta un desempeño nada favorable.

Constantemente se busca realizar modificaciones dentro del sistema educativo que permitan atacar los diferentes problemas que tanto alumnos como maestros presentan con el fin de mejorar la calidad educativa y despuntar en las evaluaciones nacionales e internacionales; sin embargo, la mejora en los resultados no se ha logrado, aquí un ejemplo. De acuerdo a la evaluación realizada por el Programa Internacional para la Evaluación de Estudiantes (PISA) en el año 2012, llevado a cabo por la Organización para la Cooperación y el Desarrollo Económico

1 Universidad Autónoma de Zacatecas. Unidad Académica de Psicología, Campus Jalpa. 
(OCDE), en el aspecto de lectura, México presenta que $41 \%$ de los alumnos mexicanos no alcanza el nivel de competencias básico y menos de $0.5 \%$ de los alumnos mexicanos de 15 años logra alcanzar los niveles de competencia más altos.

Generalmente, se justifican los resultados anteriores con un desempeño inadecuado del docente y una de las razones principales que se han utilizado es que existe un reconocido fracaso en los maestros al momento de aplicar estrategias, técnicas o métodos, aunque poco se ha rastreado el origen directo en la calidad de los procesos formativos de los profesores. Se ha observado que, aunque en la superficie el discurso de los profesores cambia, no se evidencian cambios en su práctica o, en su defecto, éstos no parecen ser profundos.

La formación del docente le permite conocer y aplicar diversas estrategias en su práctica profesional que le lleven a mejorar en este aspecto y, por consiguiente mejore el desempeño de sus alumnos.

Una vez analizado lo anterior, la presente investigación busca encontrar si existe relación entre la preparación profesional de los docentes de educación primaria del municipio de Jalpa, Zacatecas, la pertinencia de las estrategias de comprensión lectora aplicadas a sus alumnos que tanto conoce y si aplica estrategias de comprensión lectora de acuerdo al nivel de su formación profesional.

\section{Problema}

Ante este panorama, sobresale un aspecto necesario para que se pueda atacar el problema mencionado: la formación del docente vista más allá de la formación inicial. El presente trabajo se centra en la formación del maestro pero a partir de su práctica y el nivel educativo que adquiere a lo largo del tiempo, ya que desde la perspectiva de esta investigación, problemas tan cotidianos como la ineficiente comprensión lectora en los alumnos puede estar influyendo en la falta de preparación continua del profesor. 
De acuerdo con Vargas (2013), la mayoría de los docentes mexicanos cuenta con una licenciatura, la cual no necesariamente está relacionada con el nivel educativo en el que dan clases; $60.5 \%$ del total de maestros en el Sistema Educativo Nacional cuenta con un título universitario; 8.9\% de los docentes nacionales cuenta con un posgrado (maestría o doctorado); 23.3\% egresaron del sistema de escuelas normales; $3.7 \%$ sólo concluyó el bachillerato; $3.2 \%$ tiene otros estudios técnicos y 0.3\% sólo terminó la educación básica SEP (Sistema Nacional de Información Estadística Educativa, 2012).

Una vez expuesto lo anterior, surge la necesidad de identificar si existe alguna relación entre el nivel educativo del docente y la pertinencia en la aplicación de estrategias de comprensión lectora que aplica con sus alumnos, así como qué tanto se refleja en el desempeño del alumno el que el docente esté formado en niveles educativos superiores. ¿Será la formación profesional en maestría y doctorado de los docentes una manera de mejorar el desempeño de sus alumnos? ¿Será que el mayor conocimiento del docente, en específico dentro del área de la comprensión lectora, es lo que le permitirá aplicar las estrategias necesarias para que los alumnos realmente comprendan lo que leen?

\section{Método}

Participantes

Se contó con la participación de 10 escuelas de educación primaria seleccionadas por la Secretaria de Educación y Cultura (SEC) de manera azarosa, pertenecientes a las zonas escolares 19 y 20 de Jalpa, Zacatecas, y con un total de 57 docentes entrevistados.

\section{Materiales}

A través de dos instrumentos fue como se llevó a cabo la recolección de datos. Primeramente, se realizó una entrevista 
semiestructurada que constó de 13 preguntas. Mientras que el segundo instrumento fue un cuestionario de siete preguntas (total: 20 ítems). La elaboración de estos instrumentos se basó en una propuesta realizada por el INEE (2007).

\section{Procedimiento}

Primeramente, se capacitó a ocho elementos del equipo para que apoyaran en la aplicación de los instrumentos. Una vez recibidos en las escuelas, se procedió a la entrevista de manera personal y, al finalizar, se le entregaba al docente el cuestionario, el cual contestó de manera privada.

\section{Resultados}

El análisis de resultados se llevó a cabo con el programa spss versión 15 y se llegó a lo siguiente: con respecto al nivel educativo de los docentes encuestados, se observa que de los 57 docentes entrevistados, 14 de ellos estudió la Normal básica; 27 docentes, licenciatura; 12 poseen un título de naestría y sólo 4 estudiaron un doctorado.

Como se puede observar en los resultados anteriormente descritos, el mayor porcentaje recae en los docentes que estudiaron una licenciatura, con $47.4 \%$, y el menor porcentaje lo tienen los docentes con doctorado, con 7\%. De acuerdo a la perspectiva de la presente investigación, la pertinencia en la aplicación de estrategias de comprensión lectora del docente se relaciona con su nivel de estudios y se observa que son escasos los docentes con grados de estudios más allá de la licenciatura.

A continuación, se describen los resultados obtenidos al respecto de la práctica docente.

En lo que atañe a las estrategias de comprensión lectora que conocen, la mayoría de las respuestas muestra que es la estrategia de cuestionario la más conocida. De igual forma, es la más utilizada; sin embargo, el cuestionario se encuentra dentro de las estrategias procedimentales que no cumplen con la fun- 
ción de llevar al lector a la comprensión lectora a nivel profundo. Es decir, esta dinámica lleva al alumno a memorizar, rescatar ideas literales, identificar información en general, pero no lo lleva a relacionar diferentes hechos a lo largo del texto, hacer inferencias, ni tampoco a reestructurar sus conocimientos previos.

La relación encontrada con su nivel educativo se puede centrar en el hecho de que, aunque la mayoría de los docentes con los que se trabajó estudió el nivel licenciatura, sólo se obtuvieron seis respuestas referentes a estrategias de comprensión lectora de tipo comprensivas (esquemas, cuadros sinópticos, entre otros). Por lo tanto, únicamente $20.68 \%$ de respuestas son acordes a la pregunta.

De igual forma, se debe aclarar que a los docentes se les cuestionó sobre cuáles estrategias de comprensión lectora aplican en el aula para conocer si las estrategias que conocen y mencionan son las mismas que aplican. A este respecto, se encontró que de 21 respuestas obtenidas, sólo siete corresponden a estrategias comprensivas, ante esto se puede desprender que el docente conoce poco de estrategias lectoras.

De manera general se observa que la estrategia de comprensión lectora más utilizada con los alumnos es el cuestionario, la cual es una estrategia que, como ya se mencionó, no permite que el lector se adentre en la comprensión del texto, el cual es el objetivo principal al aplicar esas estrategias.

Lo anterior se relaciona con el nivel educativo de la misma forma que el análisis anterior, pues debido a que la mayoría de los docentes ha estudiado una licenciatura, se esperaría que aplicara estrategias que no sólo le permitan al lector llegar a la comprensión superficial del texto; sin embargo, esto no sucede.

\section{Discusión}

Con respecto al primer elemento relacionado con el nivel educativo de los docentes, se encontró que la mayoría poseía el grado de licenciatura, seguido de los maestros con estudios de normal Superior, después se encuentran los docentes con nivel 
de maestría y, por último, sólo cuatro docentes poseían nivel doctorado. Dentro de este aspecto es importante señalar que de acuerdo con Caldera (2010), entre más estudios posea el docente, le será más fácil acrecentar las herramientas didácticas y así poseer más estrategias para llegar a sus alumnos y, por lo tanto, tener más habilidad para aplicar en el aula estrategias pedagógicas que repercutan en una mayor calidad educativa.

En cuanto a estrategias de comprensión lectora que conoce y utiliza el docente en el aula, en ambos casos la estrategia del cuestionario es la respuesta más socorrida. En este sentido, podemos considerar que la mayoría de los docentes creen que esta estrategia procedimental lleva al alumno a la comprensión lectora; sin embargo, esto no es así, pues sólo le permite procesar información superficial, pero no lo lleva a una comprensión lectora profunda.

Con respecto al conocimiento y la pertinencia al aplicar esas estrategias, se observa que sí mencionan diversas estrategias; sin embargo, no son las mismas que aplican directamente en el aula, lo que expresa que posiblemente poseen el conocimiento sobre las estrategias, pero quizá no saben cómo llevarlas a cabo en el salón de clases, situación que se puede solventar si el docente se sigue preparando académicamente, de modo que a mayor conocimiento es más factible que en realidad lleve al alumno a cumplir el objetivo de comprender lo que lee de manera profunda.

De igual forma, se observó que los docentes con mayor grado de estudio eran los que mejores respuestas daban; es decir, que quienes conocían y aplicaban estrategias comprensivas eran los docentes con grado de estudio de maestría y doctorado, lo cual confirma lo mencionado por Caldera (2010), que entre mayor nivel de estudios, el docente posee mayor cantidad de herramientas y conocimientos.

Una vez analizado lo anterior, se observa que la relación directa entre el nivel educativo del docente y la pertinencia en la aplicación de estrategias de comprensión lectora, es una muestra de que si los docentes avanzan en su preparación profesional, no sólo las cuestiones sobre la comprensión lectora serán 
subsanadas, sino que diversas problemáticas que se presentan en el contexto áulico pueden ser mejoradas.

\section{Referencias}

Caldera, R. (2010). Práctica pedagógica de la lectura y formación docente. Revista de Pedagogía, 31(88), 15-37.

Dávalos, M.T., Aguilar, A.Y., Vital, A.T., Lozano, G., Miramontes. S. y González-Horta, A. (2013). Propuesta diagnóstica a través del Modelo de Evaluación de la Comprensión Lectora (ECOMPLEC). En Pedro Palacios, Ma. del Carmen Farfán, Enrique Navarrete y Silvia Miramontes (Eds.). Psicología educativa: reflexión, práctica e intervención (35-60). México: Libros Universitarios.

IEESA (2012), ¿De dónde vienen y a dónde van los maestros mexicanos? La formación docente en México, 1822-2012.

Instituto Nacional para la Evaluación de la Educación (2007). Prácticas docentes para el desarrollo de la comprensión lectora en primaria. Recuperado de http://www.inee.edu. mx/index.php/publicaciones.

Nieto (2009). Análisis de las políticas para maestros de educación básica en México.

OCDE. Organización de las Naciones Unidas para la Educación, la Ciencia y la Cultura (2012). Libro de cifras. Boletín estadístico del libro en Iberoamérica. Recuperado del sitio de internet de la unESCO: www.cerlalc.org/files/tabinterno/7ad328_Libro_Cifras_Ago2012.pdf.

Partido, M. (2003). La lectura como experiencia didáctica. Recuperado de www.uv.mx/cpue/coleccion/n_2728.

Pérez, M. (2012). Prácticas docentes para el desarrollo de la comprensión lectora en primaria. Recuperado de clbe.wordpress.com/2012/11/13.

Ramos, W. (2005). La comprensión lectora en profesores de la enseñanza primaria. Recuperado de http://aufop.com.

Ruiz, G. (2012). La Reforma Integral de la Educación Básica en México (RIEB) en la educación primaria: desafíos para la 
formación docente. Recuperado de http://www.aufop.com /14/02/2014.

Secretaría de Educación Pública (2008). Programa de fomento para el libro y la lectura. Recuperado de http://lectura.dgme. sep.gob.mx/0ArchivosIndex/Mexico_Lee.pdf.

Secretaría de Educación y Cultura (2011). Análisis de resultados de la prueba ENLACE 2010. CD facilitado por la SEP del Municipio de Jalpa.

Sistema Nacional de Información Estadística Educativa. (2012). Recuperado de http://www.snie.sep.gob.mx/estadisticas_ educativas.html.

Vargas, M. (2013). ¿Cuál es el perfil de los maestros mexicanos? Recuperado de http://www.adnpolitico.com/ciudadanos/2013/05/14/cual-es-el-perfil-de-los-maestros-mexicanos.

Zorrilla Fierro, M. (2011). Resultados de México en Lectura: PISA 2012. Recuperado de http://www.inee.edu.mx/index. php/boletines-mainmenu-692/446-portadas/4922-resultados-de-mexico-en-lectura-pisa-2009. 